\title{
Co-expression of pregnane $X$ receptor and ATP-binding cassette sub-family $B$ member 1 in peripheral blood: A prospective indicator for drug resistance prediction in non-small cell lung cancer
}

\author{
QINGNUAN KONG $^{1 *}$, ZENGLEI HAN $^{1 *}$, XIAOLI ZUO ${ }^{2}$, HONGJUN WEI $^{1}$ and WEIQING HUANG ${ }^{1}$ \\ ${ }^{1}$ Department of Pathology, Qingdao Municipal Hospital, Affiliated to Medical College of Qingdao University, Qingdao, \\ Shandong 266071; ${ }^{2}$ Department of Pathology, Qingdao Hiser Hospital, Qingdao, Shandong 266011, P.R. China
}

Received January 13, 2015; Accepted February 11, 2016

DOI: $10.3892 / \mathrm{ol} .2016 .4369$

\begin{abstract}
The aim of the present study was to investigate the protein expression profiling of pregnane $X$ receptor (PXR) and ATP-binding cassette sub-family B member 1 (ABCB1; also known as MDR1 or P-gp), present in the peripheral blood mononuclear cells (PBMCs) and cancerous tissues of cases of non-small cell lung cancer (NSCLC). Furthermore, the study aimed to assess the feasibility of predicting drug resistance through the medium of PBMCs. Of the subjects included in the study, 37 were histopathologically diagnosed with NSCLC and 17 were control patients without cancer. ThinPrep liquid-based smears with cytosine were applied in the examination of the PBMCs and proved quite effective in preserving the morphology and surface antigens of the lymphocytes. Measurements of expression levels in the PBMCs and cancerous tissues were obtained by immunohistochemical means. The results showed that, with the exception of the selective PXR expression in the normal lung tissues, the two types of proteins existed extensively throughout the PBMCs, normal tissues and tumors. Among the cancer patients, prior to chemotherapy, a significant rise in $\mathrm{ABCB} 1$ expression could
\end{abstract}

Correspondence to: Ms. Weiqing Huang, Department of Pathology, Qingdao Municipal Hospital, Affiliated to Medical College of Qingdao University, 5 Donghai Middle Road, Qingdao, Shandong 266071, P.R. China

E-mail: huangweiqing_40@163.com

*Contributed equally

Abbreviations: PXR, pregnane $\mathrm{X}$ receptor; ABCB1, ATP-binding cassette sub-family B member 1; PBMCs, peripheral blood mononuclear cells; NSCLC, non-small cell lung cancer; ABCC1, ATP-binding cassette subfamily $\mathrm{C}$ member 1; NRs, nuclear receptor families; CAR, constitutive androstane receptor; MDR, multidrug resistance

Key words: pregnane $\mathrm{X}$ receptor, non-small cell lung cancer, peripheral blood mononuclear cells, ATP-binding cassette sub-family B member 1 be observed in the PBMCs, together with a similar rise in ABCB1 and PXR expression in the tumor specimens. Marked upregulation of the two proteins was detected in the PBMCs following 1 cycle of first-line chemotherapy. ABCB1 expression, correlated with PXR, persisted mostly in the PBMCs and tissue samples. When bound to and activated by ligands, PXR translocates from the cytoplasm to the nucleus of the cells. PXR subsequently binds to its DNA response elements as a heterodimer with the retinoid X receptor. A PXR translocation of moderate or low differentiation was identified in 3 cases of adenocarcinoma, which were co-expressing the two genes in the PBMCs prior to chemotherapy. During follow-up visits, tumor recurrence was observed within 3 months in 5 cases, which were characterized by PXR translocation. These findings indicate that the combined expression of PXR and ABCB1 in PBMCs may be used as a prospective indicator in diagnosis prior to histopathological diagnosis, and therefore may function as a novel biomarker for the prediction of drug resistance.

\section{Introduction}

Impressive headway has been made in chemotherapy drug development, yet non-small cell lung cancer (NSCLC) types often turn out to be resistant to the treatment, whether a given drug is administered repeatedly or not. Furthermore, drug resistance mechanisms, to a great extent, remain unclear. From the viewpoint of the transport-obstruction theory, decreased uptake and increased efflux across tumor cell membranes contribute to such resistance $(1,2)$. To be specific, certain proteins, such as ATP-binding cassette (ABC) super-family, transport drug and toxic materials increase concentration gradients, and this reverse transportation has much to do with the complex issue of drug resistance.

Previous studies have detected the expression of ABC-transporters, such as ABC sub-family B member 1 (ABCB1; also known as MDR1 or P-gp) in lung cancer, and arrived at the conclusion that $\mathrm{ABCB} 1$ overexpression or polymorphism is a vital factor behind drug resistance in lung cancer treatment $(3,4)$. For example, among lung cancer patients, the majority of drug resistance cases overexpress ABCB1 and 
$\mathrm{ABC}$ subfamily $\mathrm{C}$ member 1 (ABCC1) genes, registering an $\mathrm{ABCB} 1$ expression level of 25-43\% and an $\mathrm{ABCC} 1$ incidence of $80-100 \%$ (5-7). Another previous study has suggested that in NSCLC patients, the ABCB1 $2677 \mathrm{G}>\mathrm{T} / \mathrm{A}$ polymorphism and $2677 \mathrm{G}-3435 \mathrm{C}$ haplotype may be used as treatment response predictors to docetaxel-cisplatin chemotherapy (8). It has been shown that these proteins transport chemical compounds from the nucleus to the cytoplasm, where they are redistributed in order to maintain an intracellular concentration below the cytotoxic level (9). This unique self-protective mechanism is closely associated with the multidrug resistance (MDR) phenotype of cancer cells.

A number of studies have suggested that $\mathrm{ABC}$ transporters impede drug absorption. Such a property predicts the susceptibility of a patient and therefore highlights the necessity of individualized chemotherapy. However, it is not yet easy to convincingly elucidate the molecular mechanism of MDR, as this is limited by the current level of gene regulation. Recently, nuclear receptor (NR) families, including pregnane $\mathrm{X}$ receptor (PXR) and constitutive androstane receptor (CAR), have been proven capable of boosting MDR (10). Together with xenobiotics, such as rifampin, activated NRs, as a transcriptional regulator, can play a significant role in modulating drug transporter genes and drug-metabolizing enzymes (11). A number of xenobiotic transporters are subject to PXR regulation, including ABCB1, ABCC2, ABCC3, ABCC4, ABCC5 and breast cancer resistance protein, commonly found in hepatocytes, the kidneys and the blood brain barrier (9). It has also been reported that with the presence of the ABCB1 gene enhancer, or upon activation by various therapeutic agents, such as clotrimazole and nifedipine, PXR is likely to exercise regulation over ABCB1 gene expression (12).

Under such circumstances, demand arises for a proper surrogate marker and a simple measuring technique $(13,14)$. Solid tumor studies in general, however, are beset by the problem of blood and normal tissue contamination, and often end in positive-rate, heterogeneous conclusions. Moreover, in the majority of cases, particularly at the advanced stage, the collection and inspection of target tissue samples often become difficult.

As is well known, blood cells, functioning as mediators of the immune response and interacting with all human tissues, are relevant to the pathogenesis of numerous diseases, such as renal cell carcinoma or breast cancer. Recent studies have suggested that the peripheral blood reflects physiological and pathological changes inside the body, and may be taken as a substrate for the molecular profiling of human diseases and disease risks (15). Ban et al, by aid of flow cytometry, examined the ABCB1 profile of peripheral blood mononuclear cells (PBMCs) in epilepsy patients and found a higher basal ABCB1 level in them and in patients on high-dose medications (16). Valente et al investigated the downregulation of ABCB1 in the kidneys and PBMCs of SHR rats, and suggested that for hypertensive humans, assessing gene activity in PBMCs is a non-invasive, and therefore practical, test method (17).

With non-invasive, peripheral blood-based lab tests available, the prospect of PBMCs as an alternative for drug resistance detection has come to the fore. However, the practicality of using peripheral blood cells for predicting lung cancer multidrug resistance requires investigation. With the use of immunohistochemistry, the present study assesses ABCB1 and PXR expression in PBMCs and tissue samples obtained from lung cancer patients. The study examines post-chemotherapy ABCB1 and PXR expression, and the correlations between the two.

\section{Materials and methods}

Materials. The study was conducted in strict accordance with the stipulations of the Helsinki Declaration and was approved by the Qingdao Municipal Hospital Ethics Committee (Qingdao, China). All the subjects and their families were informed of the relevant details and signed consent forms of their own accord prior to the study. The study duration ranged between November 2011 and October 2012. The subjects consisted of 37 patients with pathologically proven lung cancer (10 females with a mean age \pm standard deviation (SD) of $51 \pm 13$ years; and 27 males with a mean age \pm SD of $56 \pm 18$ years). Of the 37 cancer patients, 24 presented with lung adenocarcinoma and 13 with lung squamous carcinoma. In the control group (11 females with a mean age \pm SD of $41 \pm 9$ years; and 16 males with a mean age \pm SD of $40 \pm 13$ years), samples of non-neoplastic lung tissue were collected from 17 subjects who underwent a lung wedge resection, either due to bullae (12 cases) or benign tumors ( 2 cases of pulmonary hamartoma and 3 of papilloma).

Samples preparation. Tissue sampling was performed through surgical resection in 31 subjects and through transbronchial fine-needle aspiration biopsy in 6 subjects. Prior to this, none of the patients had ever been administered chemotherapy or radiotherapy. The specimens were fixed in $10 \%$ buffered neutral formalin. Directly prior to and following 1 cycle of first-line chemotherapy (intravenous vinorelbine, $25 \mathrm{mg} / \mathrm{m}^{2}$ on day 8 ; intravenous cisplatin, $75-80 \mathrm{mg} / \mathrm{m}^{2}$ on day 3 ), a $3-\mathrm{ml}$ blood sample was collected from each subject and transferred into an ethylenediaminetetraacetic acid (EDTA)-containing blood collection tube. PBMCs were immediately isolated by Ficoll-Hypaque (Beijing Solarbio Science \& Technology Co., Ltd., Beijing, China) density centrifugation at 1,800 x g (5417R; Eppendorf, Hamburg, Germany).

Immunohistochemical analysis of the tissue samples. The lung cancer tissue sections and controls were fixed in $10 \%$ neutral formalin, paraffin-embedded and cut into $4-\mu \mathrm{m}$ thick slices. The slices were briefly treated with $1 \mathrm{mM}$ EDTA ( $\mathrm{pH}$ 8.0) prepared by microwave, and then with $3 \%$ hydrogen peroxidase, following $10 \mathrm{~min}$ of blocking with endogenous peroxidase. The polyclonal rabbit anti-PXR (1:100; catalog no. ab85451; Abcam, Cambridge, UK) and monoclonal rabbit anti-ABCB1 (p170/MDR-1 Ab; 1:200; catalog no. MS-660; Fuzhou Maixin Biotechnology Development Co., Ltd., Fuzhou, China) primary antibodies were added at $4^{\circ} \mathrm{C}$ overnight. Liver carcinoma tissues were used as a positive control, and normal goat immunoglobulin G (UltraSensitive ${ }^{\mathrm{TM}}$ SP kit; catalog no. KIT-9720; Fuzhou Maixin Biotechnology Development Co., Ltd.) was used instead of the primary antibody as a negative control. Secondary antibody (UltraSensitive ${ }^{\text {TM }} \mathrm{SP}$ kit) was also applied at room temperature for $30 \mathrm{~min}$. Color development was performed using 3,3'-diaminobenzidine 
Table I. Comparison of ABCB1 and PXR protein expression in the PBMCs of the NSCLC patients and control group prior to chemotherapy.

\begin{tabular}{|c|c|c|c|c|c|c|c|c|c|c|}
\hline \multirow[b]{2}{*}{ Protein } & \multicolumn{5}{|c|}{ NSCLC group } & \multicolumn{5}{|c|}{ Control group } \\
\hline & $\mathrm{ABCB} 1^{+}$ & $\mathrm{ABCB} 1^{-}$ & Sum & $\mathrm{r}$ & P-value & $\mathrm{ABCB} 1^{+}$ & $\mathrm{ABCB} 1^{-}$ & Sum & $\mathrm{r}$ & P-value \\
\hline $\mathrm{PXR}^{+}, \mathrm{n}$ & 18 & 5 & $23(62.2)$ & 0.49 & 0.03 & 3 & 8 & $11(64.7)$ & 0.12 & 0.62 \\
\hline $\mathrm{PXR}^{-}, \mathrm{n}$ & 4 & 10 & $14(37.8)$ & & & 1 & 5 & $6(35.3)$ & & \\
\hline Sum, n (\%) & $22(59.5)^{\mathrm{a}}$ & $15(40.5)$ & $37(100.0)$ & & & $4(23.5)$ & $13(76.5)$ & $17(100.0)$ & & \\
\hline
\end{tabular}

${ }^{a} \mathrm{P}=0.02$ vs. control group. ABCB1, ATP-binding cassette sub-family B member 1; PXR, pregnane X receptor; PBMCs, peripheral blood mononuclear cells; NSCLC, non-small cell lung cancer.

(Zhongshan Golden Bridge Biotechnology Co., Ltd., Beijing, China), and the hemtoxylin (Fuzhou Maixin Biotechnology Development Co., Ltd.)-stained slices were coverslipped in permount (Fuzhou Maixin Biotechnology Development Co., Ltd.).

Immunohistochemistry for PBMCs. Previously centrifuged PMBCs were suspended carefully in ThinPrep conserved solution (Becton Dickinson, Franklin Lakes, NJ, USA) for 4-6 h. Afterwards, liquid-based smears with cytospin (Ningbo Medsun Medical Co., Ltd., Ningbo, China) were prepared on the principle of the ThinPrep cytological test, and then fixed in pure acetone solution for $20 \mathrm{~min}$. Following Giemsa staining (New Biotechnology Development Co., Ltd., Fuzhou, China), prior to immunostaining, the percentage of lymphocytes was calculated through microscopic observation. The process yielded a high lymphocyte purity (96\%). Immunohistochemical analysis was conducted as aforementioned.

PXR and ABCB1 expression in PBMCs and cancerous tissues was evaluated independently by two pathologists blinded to the study details. The final score was determined as the staining intensity plus the percentage of positive cells. The scoring system was graded as follows: 0-3, negative expression; and 4-10, positive expression.

Statistical analysis. Statistical analysis was performed by means of a $\chi^{2}$ test and Fisher's exact test (SPSS software, version 17.0; SPSS Inc., Chicago, IL, USA). P<0.05 was used to denote a statistically significant difference.

\section{Results}

Pre-chemotherapy $P X R$ and $A B C B 1$ protein expression in $P B M C$ s. Results from the immunostaining demonstrated widespread PXR and ABCB1 expression in the PBMCs from the lung cancer patients (prior to and following chemotherapy) and the control group. Generally, conspicuous PXR expression was mostly found in the nucleus of the lymphocytes, while high ABCB1 expression was found on the lymphocyte membrane (Fig. 1A and B). In the control group, the positive rate of $\mathrm{ABCB} 1$ and $\mathrm{PXR}$ expression reached $23.5 \%(4 / 17)$ and $64.7 \%$ (11/17), respectively. In the lung cancer patients, positive ABCB1 and PXR protein expression increased to $59.5 \%$ (22/37) and 62.2\% (23/37), respectively (Table I). PBMCs from the lung cancer patients exhibited a significant increase in ABCB1 expression. This agreed with the outcomes from previous studies. Unexpectedly, statistical analysis indicated that significant correlations between PXR and ABCB1 expression could only be found in the PBMCs from the lung cancer patients.

The results showed that no associations were identified between PXR and ABCB1 expression in the PBMCs of the control group. It may be inferred that the similarity in PXR expression in the two groups is attributable to the multi-biological functions of PXR. As a ligand-activated transcription factor, PXR plays a vital role in drug transportation regulation, as well as in endobiotic metabolism, including glucose metabolism, androgen metabolism, vitamin metabolism and bone mineral homeostasis (18). PXR, once activated, even participates in the immune response and inflammation. Thus, broad biological and physiological implications of PXR activation may underlie its unstable expression in PBMCs, particularly when the study is based on a limited sample size.

Pre-chemotherapy $P X R$ and $A B C B 1$ protein expression in cancerous tissues. In the tissue samples collected, PXR expression was mainly located in the nucleus, perinuclear regions and cytoplasm, while $\mathrm{ABCB} 1$ was located in the cytoplasm and on the cell membrane (Fig. 1C and D). For normal lung tissues, PXR protein was mainly expressed in the bronchial epithelium and alveolar macrophages (Fig. 1E), while ABCB1 protein was expressed on alveolar epithelial cells. Each protein was expressed on the tumor cells in the cancerous tissues. In the lung cancer patients, the ABCB1 and PXR proteins were positively expressed in $64.9 \%(24 / 37)$ and $56.8 \%(21 / 37)$ of tissues, respectively, compared with $41.2 \%(7 / 17)$ and $35.3 \%(6 / 17)$ in the normal lung samples (Table II). Increased ABCB1 and MDR1 protein expression was also observed in the lung cancer tissues, although its significance could not be confirmed yet, since the results were not statistically significant. Significant correlations between PXR and ABCB1 was observed in normal lung tissues and tumor specimens. Unlike in squamous carcinoma, strong positive PXR expression could be observed in the adenocarcinoma, with a significant association with differential classification (Table III). By contrast, ABCB1 expression in cancerous tissues was not associated with cancer classification and differentiation. 
Table II. Comparison of ABCB1 and PXR protein expression in non-small cell cancer tissue samples and non-neoplasm samples.

\begin{tabular}{|c|c|c|c|c|c|c|c|c|c|c|}
\hline \multirow[b]{2}{*}{ Protein } & \multicolumn{5}{|c|}{ Cancer tissues } & \multicolumn{5}{|c|}{ Non-neoplasm samples } \\
\hline & $\mathrm{ABCB}^{+}$ & ABCB1- & Sum & $\mathrm{r}$ & P-value & $\mathrm{ABCB}^{+}$ & ABCB1- & Sum & $\mathrm{r}$ & P-value \\
\hline $\mathrm{PXR}^{+}, \mathrm{n}$ & 17 & 4 & $21(56.8)$ & 0.38 & 0.02 & 5 & 1 & $6(35.3)$ & 0.63 & 0.03 \\
\hline $\mathrm{PXR}^{-}, \mathrm{n}$ & 7 & 9 & $16(43.2)$ & & & 2 & 9 & $11(64.7)$ & & \\
\hline Sum, n (\%) & $24(64.9)$ & $13(35.1)$ & $37(100.0)$ & & & 7 (41.2) & $10(58.8)$ & $17(100.0)$ & & \\
\hline
\end{tabular}

ABCB1, ATP-binding cassette sub-family B member 1; PXR, pregnane X receptor.
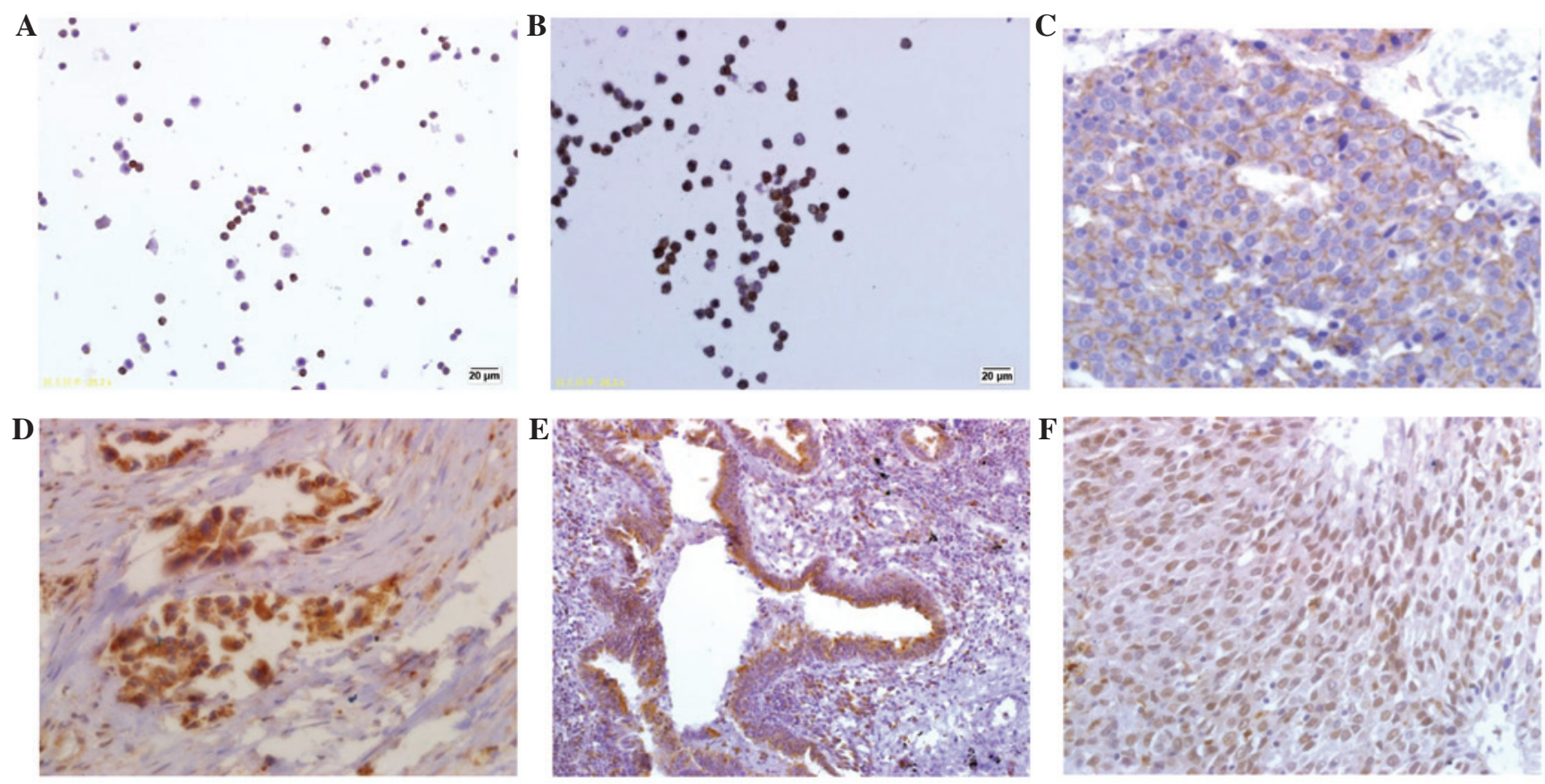

Figure 1. Representative staining of ABCB1 and PXR protein in PBMCs and tissues. (A) Representative staining of ABCB1 in PBMCs. (B) Representative staining of PXR in PBMCs. (C) Representative staining of ABCB1 in NSCLC cancer tissues. (D) Representative staining of PXR in NSCLC cancer tissues (high differentiation); expression located in the cytoplasm and perinuclear regions. (E) Representative staining of PXR expression in the bronchial epithelium of normal lung tissues. (F) Representative staining of PXR in NSCLC cancer tissues (low differentiation); expression located in the nuclei. Magnification, x200. ABCB1, ATP-binding cassette sub-family B member 1; PXR, pregnane X receptor; PBMCs, peripheral blood mononuclear cells; NSCLC, non-small cell lung cancer.

Recently, further evidence has been provided in support of the theory concerning PXR translocation in the biological process, although conflicting views persist (19). In the 3 adenocarcinoma cases (from moderate to low differentiation) in the present study, PXR protein expression was located in the nucleus and perinuclear regions; in the remaining cases, expression was located in the cell neoplasm (Fig. 1F). This phenomenon indicates that the incidence of the nuclear translocation of PXR protein may be associated with cancer classification and differentiation in NSCLC.

Correlations between $P X R$ and $A B C B 1$ protein expression in PBMCs and tissue samples. All the lung cancer patients underwent a surgical resection prior to chemotherapy. Logically, this makes it impossible to study changes in MDR1 and PXR expression triggered by chemotherapeutic agents. This poses a challenge for clinicians to investigate dynamic drug resistance in the middle of a therapeutic scheme. On the other hand, due to currently available techniques, the easy access to peripheral blood provides a solution. The present study first examined how the protein expression of PXR in PBMCs and tissue specimens was associated with that of ABCB1 prior to chemotherapy, as aforementioned. Following this, possible correlations between each prospective biomarker in the PBMCs and the counterpart tissue samples were also scrutinized (Table IV). However, no significant correlations were observed.

Post-chemotherapy PXR and ABCB1 expression and tumor recurrence. Upregulation of PXR and $\mathrm{ABCB} 1$ expression could be observed in the PBMCs of the lung cancer patients who had been administered chemotherapeutic treatment following the resection. Subsequent to 1 cycle of first-line chemotherapy, the positive PXR expression rate rose as high as $100 \%$. Post-treatment ABCB1 expression was positive in 31 cases $(83.8 \%)$ in comparison with 22 cases (59.5\%) 
prior to treatment. Tumor recurrence due to drug resistance signifies a setback for first-line chemotherapy treatment in cases where the recurrence occurs within 3-6 months of chemotherapy. In the present study, follow-up visits found a recurrence time of $<6$ months in 5 of the adenocarcinoma cases with moderate to poor differentiation. For 3 of these cases, lab results denoted uniquely nuclear expression of PXR protein. In addition, all 5 cases had previous experience of pre- and post-chemotherapy ABCB1 and PXR co-expression in the PBMCs.

Although the underlying mechanisms of PXR translocation remain undetermined, the results obtained indicate that for the prediction of drug resistance in chemotherapy, nuclear PXR expression may be an ideal indicator, and ABCB1 and PXR co-expression may be better than a single biomarker in PBMCs of NSCLC, in terms of sensitivity.

\section{Discussion}

The objective of the present study was to find a useful technical method, based on biomarker expression detection in PBMCs, for monitoring drug resistance in lung cancer chemotherapy. Previous studies have reported the correlations between PXR and $\mathrm{ABC}$ superfamily members in PBMCs and normal tissues in organs such as the liver and intestine. The present study is the first to investigate the correlations between PXR and $\mathrm{ABCB} 1$ in lung cancer cases, and to probe into the relevance of these possible biomarkers to tumor recurrence. It was found that the co-expression of $\mathrm{ABCB} 1$ and PXR protein in the PBMCs, as well as nuclear PXR expression, indicated the high incidence of tumor recurrence in NSCLC. Additionally, a liquid-based cytology technique, combined with IHC, was used to study protein expression in the peripheral blood cells.

Chemotherapy is a well-established treatment for lung cancer. However, while the solution is being distributed and metabolized in tumor cells, decreased intracellular drug concentration subtracts from the therapeutic effect. MDR is partially responsible for this predicament. Multi-biological processes and bundles of genes (ABCB1, ABCC1, lung resistance protein and excision repair cross-complementation group 1) are involved in chemotherapeutic drug resistance. Elevated expression of P-glycoprotein, encoded by the $\mathrm{ABCB} 1$ gene, has been found in lung, breast, colon and prostate cancers. The ABCB1 expression level in PBMCs could rise rapidly within $24 \mathrm{~h}$ post-administration (20) and ABCB1 mRNA expression tends to increase $\sim 7.5$-fold, induced by paclitaxel in primary NSCLC cell lines, such as the A549 cell line $(20,21)$.

Although these findings touch upon possible functions of $\mathrm{ABCB} 1$ in lung cancer chemotherapy drug resistance, in clinical practice, blockage or reversal of MDR-associated genes is rarely achieved $(12,22)$. Recent findings have revealed that certain NRs, i.e., those from the ligand-dependent NR (androgen receptor and glucocorticoid receptor) and orphan receptor (peroxisome proliferator-activated receptor, PXR and CAR) subclasses, give rise to the promoter region activation of target genes through binding to specific response elements mediated by a conserved DNA-binding domain (DBD). This provides fresh insights into the transcriptional regulation of MDR. Several previous studies, based on findings in cancer
Table III. Analysis of PXR protein expression associated with differentiation in non-small cell lung cancer patients.

\begin{tabular}{lccr}
\hline Cancer type & Cases & PXR $^{+}$ & ABCB1 \\
\hline SCC, n & & & \\
High differentiation & 5 & 1 & 3 \\
Moderate to low differentiation & 8 & 3 & 5 \\
AC, n & 6 & 4 & 5 \\
High differentiation & 18 & $13^{\text {a }}$ & 11 \\
Moderate to low differentiation & & & \\
\hline
\end{tabular}

${ }^{\mathrm{a}} \mathrm{P}<0.05$ vs. SCC. PXR, pregnane $\mathrm{X}$ receptor; SCC, squamous cell carcinoma; AC, adenocarcinoma; ABCB1, ATP-binding cassette sub-family B member 1 .

Table IV. Correlation analysis of ABCB1 and PXR in PBMCs and tissue samples.

\begin{tabular}{lcc}
\hline Correlation between & $\mathrm{r}$ & P-value \\
\hline ABCB1 in PBMCs and tissues (N) & 0.765 & 1.000 \\
ABCB1 in PBMCs and tissues (C) & 0.633 & 0.077 \\
PXR in PBMCs and tissues (N) & 0.859 & 1.000 \\
PXR in PBMCs and tissues (C) & 0.537 & 0.057 \\
\hline
\end{tabular}

ABCB1, ATP-binding cassette sub-family B member 1; PXR, pregnane $\mathrm{X}$ receptor; $\mathrm{PBMCs}$, peripheral blood mononuclear cells; $\mathrm{N}$, normal controls; $\mathrm{C}$, cancerous tissues.

cell lines and solid tumors, showed that PXR can act as a modulator to stimulate associated drug resistance markers; $\mathrm{ABCB} 1$ and anion transporter polypeptide $1 \mathrm{~A} 2$ have possible roles in tumor proliferation and apoptosis (16,17,23-25). Certain studies have indicated that PXR functions as a pivotal regulator of small molecule tyrosine kinase inhibitors, which mediate ABC-transporter protein induction in the process of drug resistance (26). Previous studies have found that various breast cancer cell lines (such as MCF-7) that are resistant to chemotherapeutic agents enhance PXR expression (25). Certain researchers believe that PXR expression is low or simply non-existent in the lung, stomach or pancreas (27). However, in the course of the present study, PXR expression was noted in the bronchial epithelium and alveolar macrophages in immunostained normal lung tissues. Furthermore, expression in the adenocarcinoma samples was prevalent, which was indicative of selective and changeable expression patterns of PXR in the process of tumorigenesis. The upregulation of PXR in adenocarcinoma, suggestive of drug resistance in clinical therapy, also pointed to the role of PXR in the mechanism of drug resistance.

Although peripheral blood is most accessible in clinical examination, gene expression in PBMCs is not the same as that in tissues. To judge whether PBMCs can be used as a suitable surrogate for the measurement of PXR and ABCB1 protein expression, parallel investigations were conducted into the expression in PBMCs and lung cancer tissues. 
Considering the multiple cell types in PBMCs, the ThinPrep cytological test was used to preserve the PBMCs, which were used as cytospin-processed, liquid-based smears to avoid expression heterogeneity that could be created by quantitative polymerase chain reaction (qPCR). Prior to the study, this procedure had only been applied in cervical, hydrothorax and ascites cytology (28). To the best of our knowledge, the present study is the first practical use of the procedure in peripheral blood protein expression detection, combined with immunohistochemical technology. It has been shown that the procedure can leave PBMC surface antigens intact. As a transcriptional factor, PXR is correlated with the ABCB1 gene in the majority of cases. However, the present study uncovered unstable PXR expression in PBMCs themselves, and came to the conclusion that PXR expression in peripheral blood is not reliable as a drug resistance predictor. In the PBMCs and tissue samples, evidence of correlations between $\mathrm{ABCB} 1$ and PXR was clear, whereas in the PBMCs of normal controls, there was no significant correlation. We hypothesize that the diverse biological functions of PBMCs in vivo may, to a certain extent, contribute to the discrepancies in PXR expression, as indicated by research findings in vitro. We suggest that the co-expression of ABCB1 and PXR in PBMCs and tissue samples, instead of the expression of either as a single marker, is more sensitive and therefore of diagnostic value to drug resistance.

A larger sample size is required to confirm this, as the supposition is only based on initial studies. At present, the role of PXR in inducing tumor cell proliferation, apoptosis and drug resistance remains controversial. According to the associated literature, PXR activation, namely higher nuclear expression of PXR, has been indicated to have an impact upon tumor prognosis, although opposing views exist $(14,29)$. The present results revealed that in the majority of cases of NSCLC, PXR expression is located in the cytoplasm and perinuclear regions, while nucleus expression is only present in adenocarcinoma cases with moderate to low differentiation. The mechanism of this viable expression patterns deserves further study. Based on the results available, we suggest that PXR expression translocation is associated with its biological function in drug resistance.

In summary, the present study demonstrated that the ABCB1 and PXR proteins are expressed in PBMCs of NSCLC patients and normal controls. This is a prerequisite factor for discussions on peripheral blood application as a surrogate material for drug resistance prediction. Furthermore, to avoid heterogeneity of the cell population in PBMCs and cancerous tissues, immunohistochemical techniques were employed to evaluate protein expression instead of qPCR. For the first time in a pathobiological examination, ThinPrep liquid-based smears were introduced for PBMC immunostaining. Unexpectedly, PXR expression in cancerous tissues appeared ubiquitous compared with the selective distribution in the normal lung tissues.

As a result of these findings, we hypothesize that the co-expression of PXR and ABCB1 in PBMCs is an ideal indicator for tumor recurrence, and that despite the initial results which require further examination, PXR translocation with tumor classification and differentiation is indicative of a specific role in drug resistance.

\section{Acknowledgements}

The present study was supported by grants from the Science and Development Foundation of Qingdao, Shandong, China (no. 11-2-Technology 3-2-(3)-nsh).

\section{References}

1. Nishio K, Nakamura T, Koh Y, Suzuki T, Fukumoto H and Saijo N: Drug resistance in lung cancer. Curr Opin Oncol 11: 109-115, 1999.

2. Gottesman MM, Fojo T and Bates SE: Multidrug resistance in cancer: Role of ATP-dependent transporters. Nat Rev Cancer 2: 48-58, 2002.

3. Ikuta K, Takemura K, Sasaki K, Kihara M, Nishimura M, Ueda N, Naito S, Lee E, Shimizu E and Yamauchi A: Expression of multidrug resistance proteins and accumulation of cisplatin in human non-small cell lung cancer cells. Biol Pharm Bull 28: 707-712, 2005.

4. Triller N, Korosec P, Kern I, Kosnik M and Debeljak A: Multidrug resistance in small cell lung cancer: Expression of P-glycoprotein, multidrug resistance protein 1 and lung resistance protein in chemo-naive patients and in relapsed disease. Lung Cancer 54: 235-240, 2006

5. Young LC, Campling BG, Voskoglou-Nomikos T, Cole SP, Deeley RG and Gerlach JH: Expression of multidrug resistance protein-related genes in lung cancer: Correlation with drug response. Clin Cancer Res 5: 673-680, 1999.

6. Paredes Lario A, Blanco García C, Echenique Elizondo M and Lobo C: Expression of proteins associated with multidrug resistance and resistance to chemotherapy in lung cancer. Arch Bronconeumol 43: 479-484, 2007 (In Spanish).

7. Galimberti S, Marchetti A, Buttitta F, Carnicelli V, Pellegrini S, Bevilacqua $G$ and Petrini M: Multidrug resistance related genes and p53 expression in human non small cell lung cancer. Anticancer Res 18: 2973-2976, 1998.

8. Pan JH, Han JX, Wu JM, Sheng LJ, Huang HN and Yu QZ: MDR1 single nucleotide polymorphisms predict response to vinorelbine-based chemotherapy in patients with non-small cell lung cancer. Respiration 75: 380-385, 2008.

9. Chen Y, Tang Y, Guo C, Wang J, Boral D and Nie D: Nuclear receptors in the multidrug resistance through the regulation of drug-metabolizing enzymes and transporters. Biochemical Pharmacology 83: 1112-1126, 2012.

10. Harmsen S, Meijerman I, Beijnen JH and Schellens JH: The role of nuclear receptors in pharmacokinetic drug-drug interactions in oncology. Cancer Treat Rev 33: 369-380, 2007.

11. Omiecinski CJ, Vanden Heuvel JP, Perdew GH and Peters JM: Xenobiotic metabolism, disposition and regulation by receptors: From biochemical phenomenon to predictors of major toxicities. Toxicol Sci 120 (Suppl 1): S49-S75, 2011.

12. Geick A, Eichelbaum M and Burk O: Nuclear receptor response elements mediate induction of intestinal MDR1 by rifampin. J Biol Chem 276: 14581-14587, 2001.

13. Hernandez-Yanez M, Heymach JV and Zurita AJ: Circulating biomarkers in advanced renal cell carcinoma: Clinical applications. Curr Oncol Rep 14: 221-229, 2012.

14. Pondugula SR and Mani S:Pregnane xenobiotic receptor in cancer pathogenesis and therapeutic response. Cancer Lett 328: $1-9,2013$.

15. Mohr S and Liew CC: The peripheral-blood transcriptome: New insights into disease and risk assessment. Trends Mol Med 13: 422-432, 2007.

16. Ban JJ, Jung KH, Chu K, Lee ST, Jeon D, Park KI, Moon HJ, Kim H, Kim S, Lee SK and Roh JK: Profiles of multidrug resistance protein-1 in the peripheral blood mononuclear cells of patients with refractory epilepsy. PLoS One 7: e36985, 2012.

17. Valente RC, Capella LS, Nascimento CR, Braga F, Echevarria-Lima J, Lopes AG and Capella MA: ABCB1 (P-glycoprotein) but not ABCC1 (MRP1) is downregulated in peripheral blood mononuclear cells of spontaneously hypertensive rats. Pflugers Arch 456: 359-368, 2008.

18. Helsley RN, Sui Y, Ai N, Park SH, Welsh WJ and Zhou C: Pregnane $\mathrm{X}$ eeceptor mediates dyslipidemia induced by the HIV protease inhibitor amprenavir in mice. Mol Pharmacol 83: 1190-1199, 2013. 
19. Godoy P, Hewitt NJ, Albrecht U, Andersen ME, Ansari N, Bhattacharya S, Bode JG, Bolleyn J, Borner C, Böttger J, et al: Recent advances in 2D and 3D in vitro systems using primary hepatocytes, alternative hepatocyte sources and non-parenchymal liver cells and their use in investigating mechanisms of hepatotoxicity, cell signaling and ADME. Arch Toxicol 87: 1315-1530, 2013.

20. Melguizo C, Prados J, Luque R, Ortiz R, Rama AR, Caba O, Rodríguez-Serrano F, Álvarez PJ and Aránega A: Modulation of multidrug resistance gene expression in peripheral blood mononuclear cells of lung cancer patients and evaluation of their clinical significance. Cancer Chemother Pharmacol 71: 537-541, 2013.

21. Melguizo C, Prados J, Luque R, Ortiz R, Caba O, Alvarez PJ, Gonzalez B and Aranega A: Modulation of MDR1 and MRP3 gene expression in lung cancer cells after paclitaxel and carboplatin exposure. Int J Mol Sci 13: 16624-16635, 2012.

22. Synold TW, Dussalt I and Forman BM: The orphan nuclear SXR coordinately regulates drug metabolism and efflux. Nat Med 7: 584-590, 2001.

23. Prados J, Melguizo C, Ortiz R, Perazzoli G, Cabeza L, Alvarez PJ, Rodriguez-Serrano F and Aranega A: Colon cancer therapy: Recent developments in nanomedicine to improve the efficacy of conventional chemotherapeutic drugs. Anticancer Agents Med Chem 13: 1204-1216, 2013.
24. Prados J, Melguizo C, Roldan H, Alvarez PJ, Ortiz R, Arias JL and Aranega A: RNA interference in the treatment of colon cancer. BioDrugs 27: 317-327, 2013.

25. Clares B, Biedma-Ortiz RA, Sáez-Fernández E, Prados JC, Melguizo C, Cabeza L, Ortiz R and Arias JL: Nano-engineering of 5-fluorouracil-loaded magnetoliposomes for combined hyperthermia and chemotherapy against colon cancer. Eur J Pharm Biopharm 85: 329-338, 2013.

26. Harmsen S, Meijerman I, Maas-Bakker RF, Beijnen JH and Schellens JH: PXR-mediated P-glycoprotein induction by small molecule tyrosine kinase inhibitors. Eur J Pharm Sci 48: 644-649, 2013.

27. Qiao E, Ji M, Wu J, Ma R, Zhang X, He Y, Zha Q, Song X, Zhu LW and Tang J: Expression of the PXR gene in various types of cancer and drug resistance. Oncol Lett 5: 1093-1100, 2013.

28. Hoda RS: Non-gynecologic cytology on liquid-based preparations: A morphologic review of facts and artifacts. Diagn Cytopathol 35: 621-634, 2007.

29. Martín-Banderas L, Sáez-Fernández E, Holgado MÁ, Durán-Lobato MM, Prados JC, Melguizo C and Arias JL: Biocompatible gemcitabine-based nanomedicine engineered by flow focusing for efficient antitumor activity. Int J Pharm 443: 103-109, 2013. 